\title{
Geometry and diameter dependence of the electronic and physical properties of GaN nanowires from first principles
}

\author{
Damien J. Carter,,${ }^{1, *}$ Julian D. Gale, ${ }^{2}$ Bernard Delley, ${ }^{3}$ and Catherine Stampfl ${ }^{1}$ \\ ${ }^{1}$ School of Physics, The University of Sydney, Sydney, New South Wales 2006, Australia \\ ${ }^{2}$ Nanochemistry Research Institute, Curtin University of Technology, GPO Box U1987, Perth 6845, Australia \\ ${ }^{3}$ Paul Scherrer Institut, $\mathrm{CH}-5232$ Villigen PSI, Switzerland \\ (Received 26 September 2007; revised manuscript received 10 January 2008; published 26 March 2008)
}

\begin{abstract}
We present a comprehensive first-principles investigation of the atomic and electronic structures of gallium nitride nanowires, and examine the dependence on nanowire diameter and shape. We consider nanowires in the [0001] growth direction, with diameters ranging from 8 to $35 \AA$, and investigate the influence of saturating the dangling bonds at the edges of nanowires. We find that unsaturated nanowires are semiconducting and contain dangling bond states in the region of the band gap, the positions of which remain rather constant with varying diameter. Saturating the nanowires with hydrogen removes these states, and the band gap decreases with increasing nanowire diameter. For the unsaturated wires there is a considerable contraction of the Ga- $\mathrm{N}$ bond lengths at the edge of the wires of $6.0 \%-7.4 \%$, while for saturated wires it is $1.5 \%$. We also calculate the heat of formation of the nanowires and find that as the diameter of the nanowire increases, the average relative stability of the nanowire increases, as intuitively expected.
\end{abstract}

DOI: 10.1103/PhysRevB.77.115349

PACS number(s): 73.22.-f, 73.61.Ey, 71.15.Nc

\section{INTRODUCTION}

Nanotechnology has the potential to impact on every aspect of science, technology, and education, with applications in areas such as semiconductor devices, integrated sensors, drug delivery systems, coatings, and structural materials, with one of the most studied nanomaterials being carbon nanotubes. ${ }^{1,2}$ Fundamental studies are required of nanostructures for a more complete understanding of their unique properties. In contrast to nanotubes, whose electronic properties are largely determined by the chirality of the nanotube, nanowires have the advantage that many of their properties, particularly electronic structure, can be controlled during growth by varying the size, composition, and growth direction. ${ }^{2}$ There have been recent reports of successful fabrication of high quality gallium nitride nanostructures such as quantum dots, nanocrystallites, nanowires, and nanotubes. ${ }^{3-5}$ Gallium nitride $(\mathrm{GaN})$ nanowires are one of the most promising building blocks in nanotechnology applications, because the large band gap and structural confinement have great potential for use in technologies related to ultraviolet-blue light-emitting diodes, detectors, lasers, high temperature and/or high power devices, and potential spintronic devices. ${ }^{6}$

Experimental studies producing GaN nanowires typically generate them with the wurtzite structure, ${ }^{6-12}$ although they have also been reported in the zinc blende structure. ${ }^{13}$ The diameters of wires synthesized experimentally typically range from approximately 5 to $100 \mathrm{~nm} \cdot{ }^{10,11}$ There are also a number of different growth directions reported, including the $[0001],{ }^{6,10-12}[10 \overline{1} 0],{ }^{14,15}$ and [11 $\left.\overline{2} 0\right]$ (Refs. 12 and 15) directions. Recently $\mathrm{GaN}$ nanowires with internal $p$ - $n$ junctions have been fabricated through $\mathrm{Mg}$ incorporation. ${ }^{16} \mathrm{GaN}$ nanowires have also recently been investigated as dilute magnetic semiconductor systems. In particular, when doped with $\mathrm{Mn}$, a ferromagnetic behavior at room temperature has been reported. ${ }^{17-19}$
There have been several recent theoretical investigations of the electronic structure of $\mathrm{GaN}$ nanowires, and in all cases, a periodic slab model was used to calculate the nanowires. Tsai et al. ${ }^{20}$ examined three unsaturated nanowires in the [0001] growth direction with diameters of 10,15 , and $18 \AA$, and found that the average bond length of the nanowire decreases as the nanowire diameter decreases. Gulans and $\mathrm{Tale}^{21}$ examined three unsaturated nanowires in the [0001] direction of approximately $20 \AA$ diameter, with shapes ranging from hexagonal to essentially circular, finding the hexagonal shaped nanowire is the most stable. They also found that Young's modulus of the nanowire decreases with decreasing diameter. Wang et al. ${ }^{22,23}$ have calculated the electronic structure of an unsaturated nanowire in the [0001] growth direction with a $10 \AA$ diameter, and examined the magnetic properties after pair doping with manganese or chromium atoms. They found that both dopants produce ferromagnetic coupling, with preferential substitution of these atoms at the edge of the nanowire.

There have also been a number of recent electronic structure calculations of other semiconductor nanowires including $\mathrm{Si}^{24-27} \mathrm{ZnO}^{28} \mathrm{AlN}^{29}{ }^{29} \mathrm{GaAs},{ }^{30}$ and $\mathrm{InP}^{30,31}$ Zheng et al. ${ }^{25}$ reported that the band gap of hydrogen-saturated $\mathrm{Si}$ [001] nanowires decreases with increasing diameter, over the diameter range of 7-27 $\AA$. Zhao et al. ${ }^{29}$ found that the strain energy of unsaturated AlN [0001] nanowires is inversely proportional to the nanowire diameter, for nanowires with diameters ranging from 7 to $22 \AA$. Schmidt et al. ${ }^{31}$ investigated hydrogen-saturated InP [111] nanowires with diameters ranging from 18 to $21 \AA$, and reported that the energy gap is proportional to $1 / d^{2}$ (where $d$ is the diameter).

To date there has been no comprehensive investigation into the atomic and electronic structures of $\mathrm{GaN}$ nanowires and their dependence on diameter and geometry, nor the effect of saturation of dangling bonds. The present work represents an extensive study of these systems, which we perform using first-principles density functional theory calculations. 


\section{METHODOLOGY}

Density functional theory (DFT) calculations are performed using two codes, namely, SIESTA (Refs. 32 and 33) and $\mathrm{DMOL}^{3}, 3,34,35$ where we employ the generalized gradient approximation (GGA) of Perdew et al. ${ }^{36}$ For SIESTA, the norm-conserving pseudopotentials of Troullier and Martins ${ }^{37}$ are used, with the valence electron configurations of gallium $3 d^{10}, 4 s^{2}, 4 p^{1}$, nitrogen $2 s^{2}, 2 p^{3}$, and hydrogen $1 s^{1}$. A double zeta basis set with polarization functions is used for all atoms. The localized basis set in SIESTA consists of numerical atomic orbitals, which are radially confined to an extent that induces an energy shift in each orbital of $0.01 \mathrm{Ry}$. Hartree and exchange-correlation energies are evaluated on a uniform real-space grid of points with a defined maximum kinetic energy of $300 \mathrm{Ry}$. The Brillouin zone of the bulk is sampled using an $(8 \times 8 \times 5)$ Monkhorst-Pack ${ }^{38} \mathbf{k}$-grid generating $160 \mathbf{k}$ points in the irreducible part of the Brillouin zone (IBZ), and for all nanowires, a $(1 \times 1 \times 3) \mathbf{k}$ grid is used, producing $2 \mathbf{k}$ points in the IBZ. For $\mathrm{DMOL}^{3}$, core electrons are represented by the semilocal density functional semicore pseudopotential (DSPP), ${ }^{39}$ with the valence electron configurations of gallium $3 d^{10}, 4 s^{2}, 4 p^{1}$, nitrogen $1 s^{2}$, $2 s^{2}, 2 p^{3}$, and hydrogen $1 s^{1}$. A double numerical basis set with polarization functions is used for all atoms, using a real-space cutoff of 9 bohr. The Brillouin zone of the bulk is sampled using a $(6 \times 6 \times 6)$ Monkhorst-Pack ${ }^{38} \mathbf{k}$-grid generating $21 \mathbf{k}$ points in the IBZ. Nanowires are sampled with a $(2 \times 2 \times 2) \mathbf{k}$ grid, producing $2 \mathbf{k}$ points and $4 \mathbf{k}$ points in the IBZ for hexagonal and triangular cross-section nanowires, respectively. We checked the convergence of the real-space cutoff by considering values of 5, 7, 9, and 11 bohr. For both the wurtzite and zinc blende structures, the energy difference when changing from a cutoff of 9 to $11 \mathrm{bohr}$, is less than 1 mhartree. This indicates that the real-space cutoff is suitably converged using a value of 9 bohr. Similar convergence checks were carried out with SIESTA. We checked the convergence with respect to $\mathbf{k}$ points, by using $(6 \times 6 \times 6),(8 \times 8$ $\times 8),(10 \times 10 \times 10)$, and $(12 \times 12 \times 12)$ Monkhorst-Pack k grids, for both the zinc blende and wurtzite structures, and the energy difference between all different grid choices is less than 1 mhartree. Similar convergence tests were also carried out with SIESTA, giving us confidence our structures are highly converged with respect to $\mathbf{k}$ points.

The GDIS program ${ }^{40}$ is used to generate nanowires in the [0001] growth direction. Different shape and size nanowires are chosen in such a way as to minimize the number of dangling bonds on outer edge atoms. We define the nanowire diameter as the maximum distance between edge atoms on opposite sides of the nanowire. Full atomic relaxation is performed for all nanowires with both codes. These calculations are also carried out with dangling bonds on nanowires saturated with hydrogen atoms (both SIESTA and $\mathrm{DMOL}^{3}$ ) and appropriate fractionally charged hydrogen atoms ( $\mathrm{DMOL}^{3}$ only).

\section{RESULTS AND DISCUSSION}

\section{A. Bulk $a-\mathrm{Ga}, \mathrm{GaN}$, and the $\mathrm{N}_{2}$ molecule}

We first calculate the physical properties of the $\mathrm{N}_{2}$ dimer, bulk $a-\mathrm{Ga}$ and $\mathrm{GaN}$, in order to examine the accuracy of
TABLE I. Calculated and experimental physical properties of the $\mathrm{N}_{2}$ dimer. PPPW represents pseudopotential plane-wave calculations.

\begin{tabular}{lcccc}
\hline \hline & SIESTA & DMOL $^{3}$ & Expt. $^{\mathrm{a}}$ & PPPW $^{\mathrm{b}}$ \\
\hline Bond length $(\AA)$ & 1.124 & 1.106 & 1.098 & 1.105 \\
Frequency $\left(\mathrm{cm}^{-1}\right)$ & 2282 & 2359 & 2359 & 2346 \\
Binding energy $(\mathrm{eV})$ & 9.55 & 10.34 & 9.75 & 9.87 \\
\hline
\end{tabular}

${ }^{\mathrm{a}}$ Reference 41.

${ }^{\mathrm{b}}$ Reference 42 .

numerical aspects of SIESTA and DMOL ${ }^{3}$ calculations. The results for $\mathrm{N}_{2}$ and $a$-Ga are listed in Tables I and II, respectively. For calculations of $a$-Ga we use the same SIESTA and $\mathrm{DMOL}^{3}$ parameters as for $\mathrm{GaN}$ calculations. However, for calculations of the $\mathrm{N}_{2}$ dimer and the isolated $\mathrm{Ga}$ atom, we use slightly more accurate parameters, with SIESTA using an energy shift of 0.005 Ry and maximum kinetic energy cutoff of $400 \mathrm{Ry}$, and $\mathrm{DMOL}^{3}$ using a real-space cutoff of $20 \mathrm{bohr}$.

For the $\mathrm{N}_{2}$ dimer, the bond length calculated with SIESTA is $2.3 \%$ larger than experiment whereas the $\mathrm{DMOL}^{3}$ bond length is only $0.7 \%$ larger. Correspondingly, with the longer bond length for the dimer, the vibrational frequency calculated with SIESTA is somewhat smaller, when compared to the $\mathrm{DMOL}^{3}$ and experimental values. The binding energies for SIESTA and DMOL ${ }^{3}$ vary by about $0.8 \mathrm{eV}$, with values of 9.55 and $10.34 \mathrm{eV}$, respectively. SIESTA calculations employ the Trouiller-Martins ${ }^{37}$ pseudopotential formulation while for DMOL $^{3}$ we use the DSPP, ${ }^{39}$ so the different pseudopotentials may explain the differences in binding energies. The pseudopotential plane-wave (PPPW) calculations of Stampfl and Van de Walle ${ }^{42}$ also use Trouiller-Martins pseudopotentials, and report a binding energy of $9.87 \mathrm{eV}$, much closer in agreement to the SIESTA value. For the orthorhombic $\left(D_{2 \mathrm{~h}}^{18}\right.$ space group) $a$-Ga structure, the calculated lattice parameter, $a$, with SIESTA is approximately $3.9 \%$ larger than experiment, and $2.6 \%$ larger with $\mathrm{DMOL}^{3}$. The calculated cohesive energies for SIESTA and $\mathrm{DMOL}^{3}$ compare closely with previous results from Fuchs et $a l^{45}$ and are only slightly underestimated with respect to experiment $(2.5 \%$ less with SIESTA and $3.9 \%$ less with $\mathrm{DMOL}^{3}$ ). All calculated bulk moduli values in Table II are close to the experimental values, or slightly larger in the case of SIESTA. As all calculations report larger

TABLE II. Calculated and experimental physical properties of bulk $a$-Ga. PPPW represents pseudopotential plane-wave calculations.

\begin{tabular}{lcccc}
\hline \hline & SIESTA & DMOL $^{3}$ & Expt. & PPPW $^{\mathrm{a}}$ \\
\hline$a(\AA)$ & 4.704 & 4.645 & $4.526^{\mathrm{b}}$ & 4.590 \\
$c / a$ & 1.660 & 1.671 & 1.692 & 1.690 \\
$b / a$ & 0.985 & 0.986 & 0.998 & 0.993 \\
Bulk modulus $(\mathrm{GPa})$ & 71 & 58 & $50-60^{\mathrm{c}}$ & 49 \\
Cohesive energy $(\mathrm{eV})$ & 2.74 & 2.70 & $2.81^{\mathrm{c}}$ & 2.69 \\
\hline \hline
\end{tabular}

aeference 45 .

${ }^{\mathrm{b}}$ Reference 43.

${ }^{\mathrm{c}}$ Reference 44. 
TABLE III. Bulk properties of wurtzite $\mathrm{GaN}$ as calculated using DFT-GGA and comparison with experimental results. PPPW represents pseudopotential plane-wave calculations.

\begin{tabular}{lcccc}
\hline \hline & SIESTA & DMOL $^{3}$ & Expt. & PPPW $^{\mathrm{a}}$ \\
\hline$a(\AA)$ & 3.28 & 3.18 & $3.19^{\mathrm{b}}$ & 3.24 \\
$c(\AA)$ & 5.31 & 5.18 & $5.19^{\mathrm{b}}$ & 5.17 \\
$u$ & 0.378 & 0.377 & $0.377^{\mathrm{b}}$ & 0.376 \\
Bulk modulus $(\mathrm{GPa})$ & 150 & 180 & $188-245^{\mathrm{c}, \mathrm{d}}$ & 172 \\
Band gap $(\mathrm{eV})$ & 1.44 & 2.58 & $3.50^{\mathrm{e}}$ & 1.45 \\
Cohesive energy $(\mathrm{eV})$ & 8.43 & 9.06 & $9.06^{\mathrm{f}}$ & 8.26 \\
Heat of formation $(\mathrm{eV})$ & -0.92 & -1.19 & $-1.14^{\mathrm{g}}$ & \\
\hline \hline
\end{tabular}

${ }^{\mathrm{a}}$ Reference 42 .

${ }^{b}$ Reference 48 .

${ }^{\mathrm{c}}$ Reference 49.

${ }^{\mathrm{d}}$ Reference 50.

eReference 51.

${ }^{\mathrm{f}}$ Reference 52 .

${ }^{g}$ Reference 53.

lattice parameters than the experimental values, one might expect that the corresponding bulk moduli values should be less than the experimental value. However, this last observation is a generalization that typically applies to simple ionic solids, whereas the $a$-Ga structure is a particularly complex structure where both covalent and metallic bonds coexist. ${ }^{46}$ Improvement in the SIESTA results can be obtained through the optimization of the basis set with respect to the material of interest; ${ }^{47}$ however, this can reduce transferability.

The optimized bulk structures of wurtzite and zinc blende GaN are listed in Tables III and IV, respectively. DMOL ${ }^{3}$ produces lattice parameters, cohesive energies, heats of formation, and bulk moduli values that are in very close agreement with experimental values. The lattice parameters from SIESTA calculations are 2\% $3 \%$ larger than the experimental values, consistent with observations that GGA functionals can overestimate cell volumes by a few percent. ${ }^{56}$ Other bulk properties calculated with SIESTA also show reasonable agreement with those of other calculations and experimental studies. The SIESTA results compare well with the DFT calculations

TABLE IV. Bulk properties of zinc blende GaN as calculated using DFT-GGA and comparison with experimental results. PPPW represents pseudopotential plane-wave calculations.

\begin{tabular}{lcccc}
\hline \hline & SIESTA & DMOL $^{3}$ & Expt. & PPPW $^{\mathrm{a}}$ \\
\hline$a(\AA)$ & 4.58 & 4.50 & $4.50^{\mathrm{b}}$ & 4.59 \\
Bulk modulus $(\mathrm{GPa})$ & 151 & 177 & $190^{\mathrm{b}}$ & 156 \\
Band gap $(\mathrm{eV})$ & 1.33 & 2.46 & $3.45^{\mathrm{c}}$ & 1.28 \\
Cohesive energy $(\mathrm{eV})$ & 8.40 & 9.04 & $9.06^{\mathrm{d}}$ & 8.25 \\
Heat of formation $(\mathrm{eV})$ & -0.89 & -1.17 & $-1.14^{\mathrm{e}}$ & \\
\hline \hline
\end{tabular}

${ }^{\mathrm{a}}$ Reference 42

${ }^{\mathrm{b}}$ Reference 54 .

${ }^{\mathrm{c}}$ Reference 55 .

${ }^{\mathrm{d}}$ Reference 52 .

${ }^{\mathrm{e}}$ Reference 53.
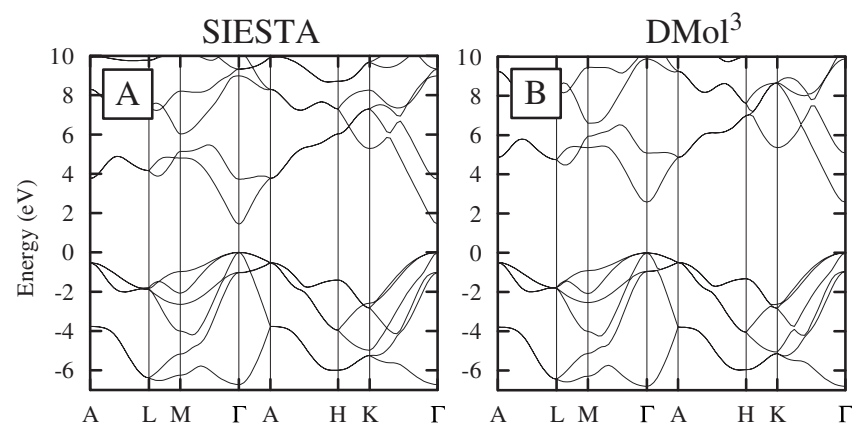

FIG. 1. Band structure of wurtzite $\mathrm{GaN}$ calculated using (A) SIESTA and (B) DMOL ${ }^{3}$.

of Stampfl and Van de Walle ${ }^{42}$ which, as mentioned above, also employ the Trouiller-Martins pseudopotential. In contrast to the binding and cohesive energies, we define the heat of formation such that a negative number indicates an exothermic process. Both calculations correctly predict the wurtzite ground state structure.

The band structures of wurtzite $\mathrm{GaN}$ as obtained from SIESTA and DMOL ${ }^{3}$ are shown in Fig. 1. Band gaps calculated using DFT are systematically underestimated when compared to experimental values. There is a noticeable difference (about $1 \mathrm{eV}$ ) between the band gaps calculated with $\mathrm{DMOL}^{3}$ and SIESTA, as reported in Tables III and IV. Band gaps can be very sensitive to the choice of functional, so the different pseudopotentials employed in the DMOL ${ }^{3}$ and SIESTA codes, together with the slightly different lattice parameters, could indeed give rise to this effect. The overall form of the band structures from SIESTA and DMOL ${ }^{3}$ in Fig. 1 match closely.

\section{B. Nanowires}

We generate wurtzite GaN nanowires in the [0001] growth direction for both hexagonal and triangular cross sections. GaN nanowires typically form hexagonal shapes,${ }^{57}$ although in the $[11 \overline{2} 0]$ direction, triangular shape nanowires have also been observed. ${ }^{58,59}$ The diameters that we study vary from approximately 8 to $35 \AA$. The shapes of nanowires are chosen in such a way as to minimize the number of dangling bonds on outer edge atoms. The resulting edge atoms have a minimum coordination of 3 , leaving at most one dangling bond on the edge atoms. We examine the atomic structure and electronic properties of both unsaturated and saturated (with hydrogen or fractionally charged hydrogen) nanowires. All nanowires considered are shown in Fig. 2. All hexagonal and triangular nanowires have $\{10 \overline{1} 0\}$ lateral facets. We selected these facets since the surface energy of GaN $(10 \overline{1} 0)$ is lower compared to the $(11 \overline{2} 0)$ surface (118 versus $123 \mathrm{meV} / \AA^{2}$, respectively). ${ }^{60}$

\section{Atomic structure}

Examining the nanowires (hexagonal and triangular) with SIESTA, when dangling bonds are not saturated, the Ga-N bond length at the outermost edge along the [0001] direction ranges from 1.858 to $1.886 \AA$ (see " $\mathrm{L}_{1}$ " in Fig. 3). Com- 
A
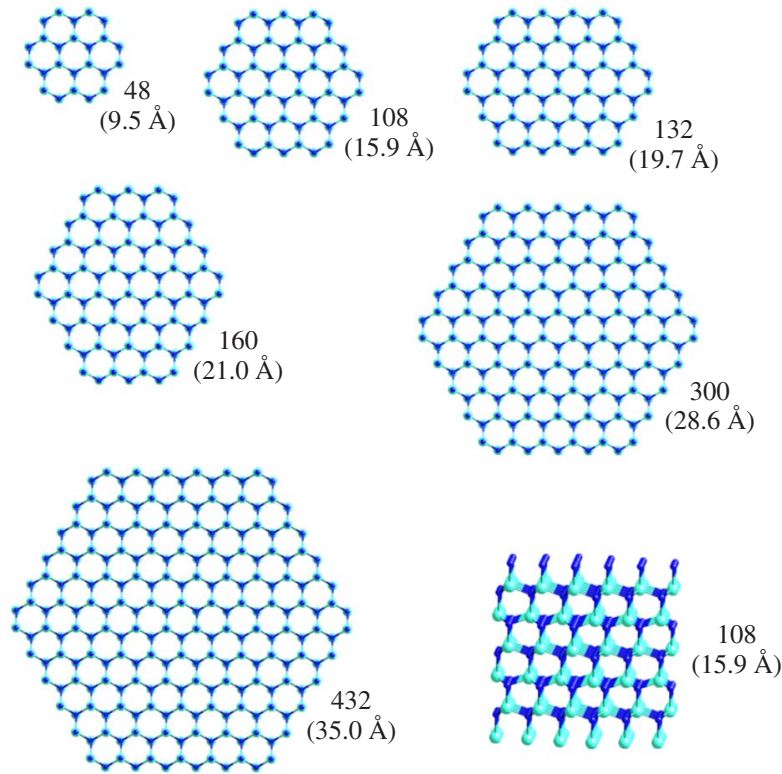

B
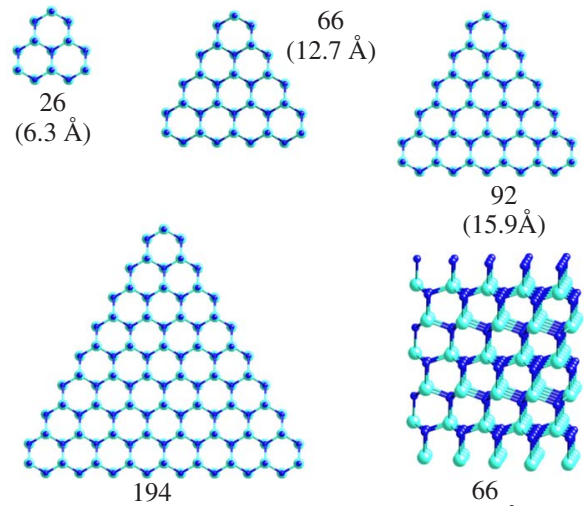

$(25.5 \AA)$
$(15.9 \AA)$

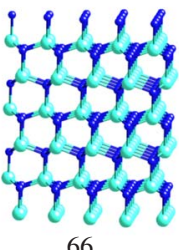

$(12.7 \AA)$

FIG. 2. (Color online) Nanowires in the [0001] direction that have (A) hexagonal and (B) triangular shaped cross sections, viewed along the wire direction, with perspective views of the 108 and 66 atom nanowires included. The number of atoms used in the calculations for each nanowire, and the diameter (in parentheses) are also labeled. Nitrogen and gallium atoms are indicated by the dark (blue) and light (aqua) spheres, respectively.

pared to the bulk value of $2.006 \AA$, this represents a contraction of $6.0 \%-7.4 \%$. This behavior is found across the entire range of diameters of nanowires examined. At the center of the nanowires, the contraction is less than $\sim 0.3 \%$. Using $\mathrm{DMOL}^{3}$, a similar contraction is also found, with values ranging from $5.9 \%$ to $6.9 \%$ for all the range of nanowires examined, and contractions within the nanowires, similarly less than $\sim 0.6 \%$. These values are close to the contractions reported for other DFT calculations, with Wang et al. ${ }^{22}$ obtaining a $5.9 \%$ contraction for a $10 \AA$ diameter nanowire, and Tsai et al..$^{20}$ obtaining a $6.2 \%-6.4 \%$ contraction for $10-18 \AA$ diameter nanowires. Theoretical calculations of the GaN

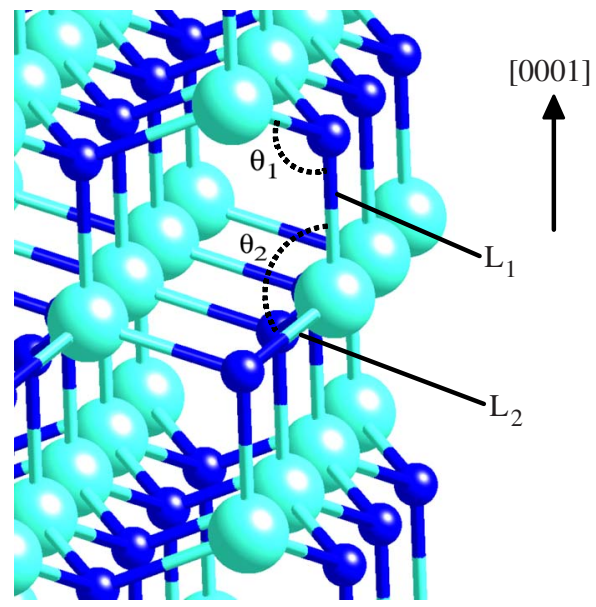

FIG. 3. (Color online) Section of a hexagonal GaN nanowire (108 atoms), illustrating the change in outer edge $\mathrm{Ga}-\mathrm{N}$ bonds and corresponding angles, where nitrogen and gallium atoms are represented by the dark (blue) and light (aqua) spheres, respectively.
(1010) surface also report a similar $\sim 6 \%$ contraction. ${ }^{60}$

The $\mathrm{Ga}-\mathrm{N}$ bond which forms a zigzag chain from the outermost surface, running perpendicular to the nanowire direction, also contracts during relaxation (see " $\mathrm{L}_{2}$ " in Fig. 3). For SIESTA, the zigzag bond length at the outermost edge of the nanowire contracts by $1.5 \%-2.5 \%$, while in the center of the nanowire, the contraction is less than $1.2 \%$. Using $\mathrm{DMOL}^{3}$, this contraction is $1.4 \%-2.8 \%$ at the outermost edge of the nanowire and less than $0.6 \%$ in the center of the nanowire. Wang et al. ${ }^{22}$ reported that the contraction of the zigzag chain at the outermost edge is $\sim 2 \%$ from calculations of a $10 \AA$ diameter nanowire, comparing well with the values from SIESTA and DMOL ${ }^{3}$ calculations.

The contraction in Ga-N bonds at the edge of the nanowires also produces a corresponding change in bond angles, with the N-Ga-N and Ga-N-Ga angles of hexagonal nanowires being $109.0^{\circ}$ before relaxation using SIESTA, changing to $113.7^{\circ}-116.6^{\circ}$ and $107.5^{\circ}-108.5^{\circ}$, respectively, after relaxation. These Ga-N-Ga $\left(\theta_{1}\right)$ and $\mathrm{N}-\mathrm{Ga}-\mathrm{N}\left(\theta_{2}\right)$ angles are illustrated in Fig. 3. Using $\mathrm{DMOL}^{3}$, similar changes were again observed with the bond angles changing from $109.1^{\circ}$ before relaxation, to $113.8^{\circ}-115.2^{\circ}$ and $106.1^{\circ}-107.7^{\circ}$, respectively, after relaxation. Similar changes were observed with both codes for triangular nanowires. Theoretical calculations of the $\mathrm{GaN}(10 \overline{1} 0)$ surface also show similar changes, with bond angles of $\sim 118^{\circ}$ and $\sim 105^{\circ}$ after relaxation. ${ }^{60}$

If we consider the average Ga-N bond length of the whole nanowire we find, for the three selected diameters of 6.3, 12.7, and $25.5 \AA$ for the triangular wires using $\mathrm{DMOL}^{3}$, the values of $1.920,1.930$, and $1.939 \AA$, respectively. For the three hexagonal wires of $9.5,15.9$, and $28.6 \AA$, we obtain $1.927,1.936$, and $1.938 \AA$, again increasing with nanowire diameter, approaching the bulk value as expected. The aver- 
Unsaturated nanowires
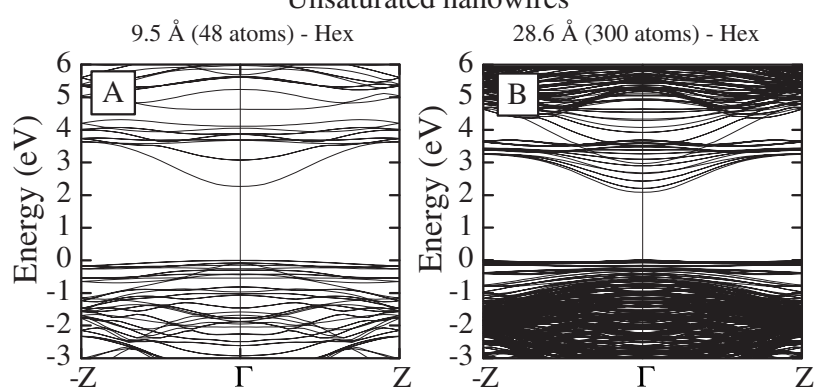

$12.7 \AA$ ( 66 atoms) - Tri
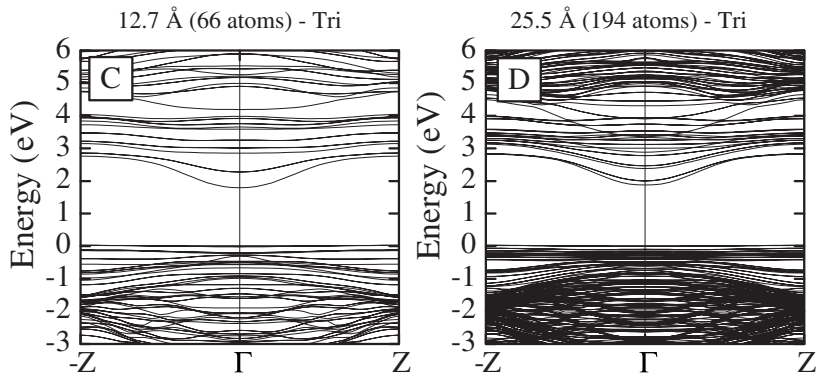

FIG. 4. The band structures for unsaturated hexagonal nanowires with diameters of (A) 9.5 (48 atoms) and (B) 28.6 (300 atoms) $\AA$, and triangular nanowires with diameters of (C) 12.7 (66 atoms) and (D) 25.5 (194 atoms) $\AA$. Calculations are performed using the $\mathrm{DMOL}^{3}$ code, and the energy zero is set at the highest occupied level. "Hex" and "Tri" represent "hexagonal" and "triangular," respectively.

age $\mathrm{Ga}-\mathrm{N}$ bond length in bulk $\mathrm{GaN}$ obtained using $\mathrm{DMOL}^{3}$ is $1.945 \AA$ A. Similar results are found using SIESTA.

After saturating the dangling bonds of the nanowires with hydrogen, there are still the same trends in the changes in the Ga-N bond lengths as described above for unsaturated nanowires, although these changes are now much less. For SIESTA, the contraction of Ga-N bonds along the [0001] nanowire range from less than $0.5 \%$ at the edge of the nanowire to less than $0.2 \%$ at the center, while for the zigzag Ga-N bonds, the contraction ranges from $0.2 \%$ to $1.5 \%$ at the edge of the nanowires to a slight expansion of less than $0.4 \%$ at the center. For $\mathrm{DMOL}^{3}$, we examined the changes for saturation with both hydrogen atoms and fractionally charged (either $0.75 e$ for $\mathrm{N}$ or $1.25 e$ for $\mathrm{Ga}$ ) hydrogen atoms. When saturated with hydrogen, the contraction of $\mathrm{Ga}-\mathrm{N}$ bonds along the [0001] nanowire range from $1.0 \%$ to $1.7 \%$ at the edge of the nanowire to less than $0.2 \%$ at the center, while for the zigzag Ga-N bonds, the contraction ranges from $0.7 \%$ to $1.0 \%$ at the edge of the nanowires to a small expansion of less than $0.4 \%$ at the center. When saturated with fractionally charged hydrogen, these respective values change to $1.1 \%-$ $1.3 \%$ (edge), less than $0.2 \%$ (center), $0.5 \%-0.9 \%$ (edge zigzag), and less than $0.2 \%$ (center zigzag).

\section{Band structure}

The band structures for unsaturated nanowires are shown in Fig. 4, as calculated by $\mathrm{DMOL}^{3}$ for hexagonal nanowires with diameters of 9.5 and $28.6 \AA$ [Figs. 4(a) and 4(b)] and triangular nanowires with diameters of 12.7 and $25.5 \AA$ [Figs. 4(c) and 4(d)] A similar result is obtained for all sized
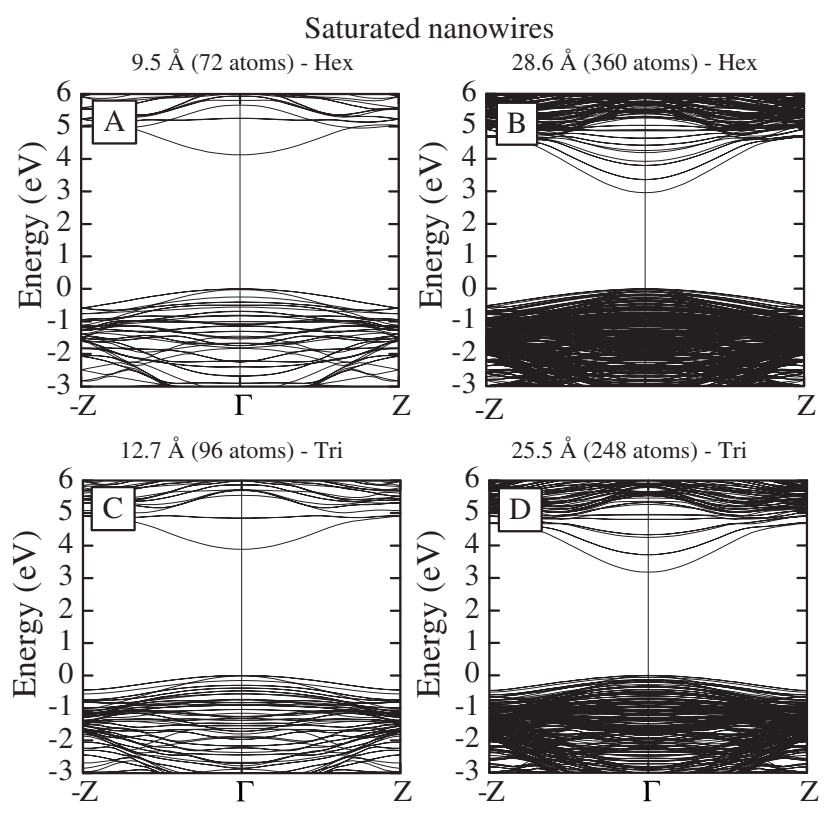

FIG. 5. The band structures for saturated hexagonal nanowires with diameters of (A) 9.5 (72 atoms) and (B) 28.6 (360 atoms) $\AA$, and triangular nanowires with diameters of (C) 12.7 (96 atoms) and (D) 25.5 (248 atoms) $\AA$. Calculations are performed using the $\mathrm{DMOL}^{3}$ code, and the energy zero is set at the highest occupied level. "Hex" and "Tri" represent "hexagonal" and "triangular," respectively.

nanowires, so we have selected one nanowire as a representative of a "small" or a "large" diameter hexagonal or triangular nanowire. Figure 5 shows the corresponding result for saturated wires. From Fig. 4, it can be seen that the "band gaps" for the unsaturated nanowires are less than for the saturated nanowires. This is due to dangling bonds of edge atoms on the unsaturated nanowires. These dangling bonds produce edge-induced states (bands) in the band gaps located above the valence band maximum and below the conduction band minimum (CBM). From comparison with the band structures of the corresponding saturated nanowire, these edge-induced states can be clearly seen, and will be described later in more detail. The band gap of unsaturated nanowires, i.e., the gap between the edge-induced states, does not change significantly with nanowire diameter since they are quite localized. When the nanowires are saturated with hydrogen, these dangling bond bands are removed from the band gap, and the band gap decreases with increasing nanowire diameter, as will be discussed in more detail below. A similar behavior has been reported for AlN nanowires. ${ }^{29}$

For the saturated and unsaturated nanowires, we investigate the spatial distribution of the electronic states in the region of the band gap, at the gamma point. For the unsaturated nanowires we find that the groups of states at the bottom and the top of the band gap have a significant weight at the edge of the nanowires and are induced by the dangling bonds. For the saturated nanowires, the states at the bottom and top of the band gap are bulklike. To illustrate this, we show in Figs. 6 and 7, the highest occupied molecular orbital (HOMO) and lowest unoccupied molecular orbital (LUMO) 

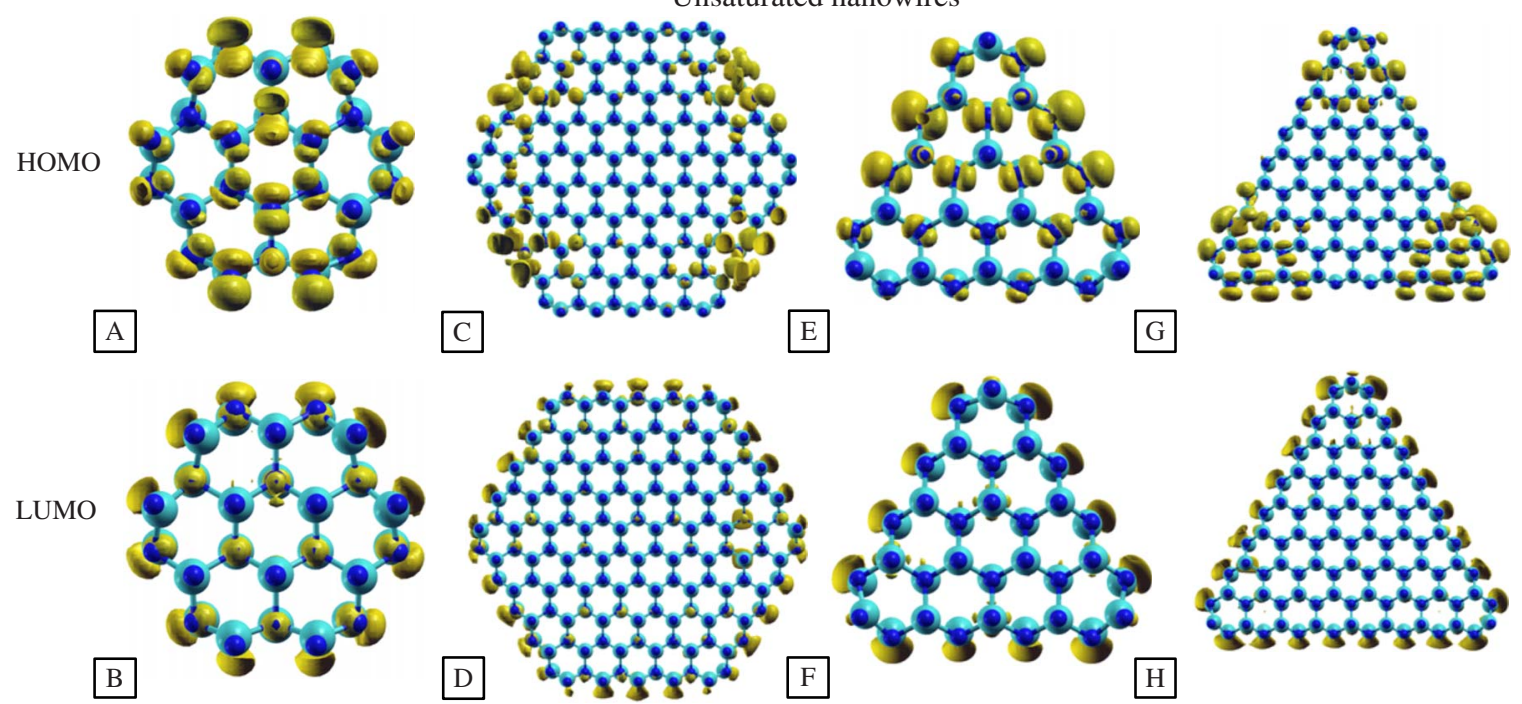

FIG. 6. (Color online) Spatial distribution of the HOMO and LUMO states at the gamma point for unsaturated nanowires, from DMOL ${ }^{3}$ calculations. The HOMO states (upper panel) are shown for hexagonal wires with diameters of (A) 9.5 (48 atoms) and (C) 28.6 (300 atoms) $\AA$, and for triangular nanowires with diameters of (E) 12.7 (66 atoms) and (G) 25.5 (194 atoms). The LUMO states (lower panel) are shown for hexagonal wires with diameters of (B) 9.5 and (D) $28.6 \AA$, and for triangular nanowires with diameters of (F) 12.7 and (H) $25.5 \AA$. Nitrogen and gallium atoms are indicated by dark (blue) and light (aqua) spheres, respectively, and the orbitals are pale gray (yellow).

states, as calculated by $\mathrm{DMOL}^{3}$, for hexagonal nanowires with diameters of 9.5 and $28.6 \AA$ and triangular nanowires with diameters of 12.7 and $25.5 \AA$, both unsaturated and saturated with hydrogen, respectively.

For the unsaturated nanowires illustrated in Fig. 6, the highest occupied and lowest unoccupied states have a significant weight at the edge of the nanowires. This is consis- tent for all nanowire diameters and for both shapes. For the smaller diameter nanowires, there can still be a visible contribution toward the center of nanowires, due to their small diameters. However, for the larger nanowires, this effect is much less. Looking closely at the HOMO states in Fig. 6, the orbital contributions appear to be centered mainly on the nitrogen atoms, with $p$ character, while the LUMO states

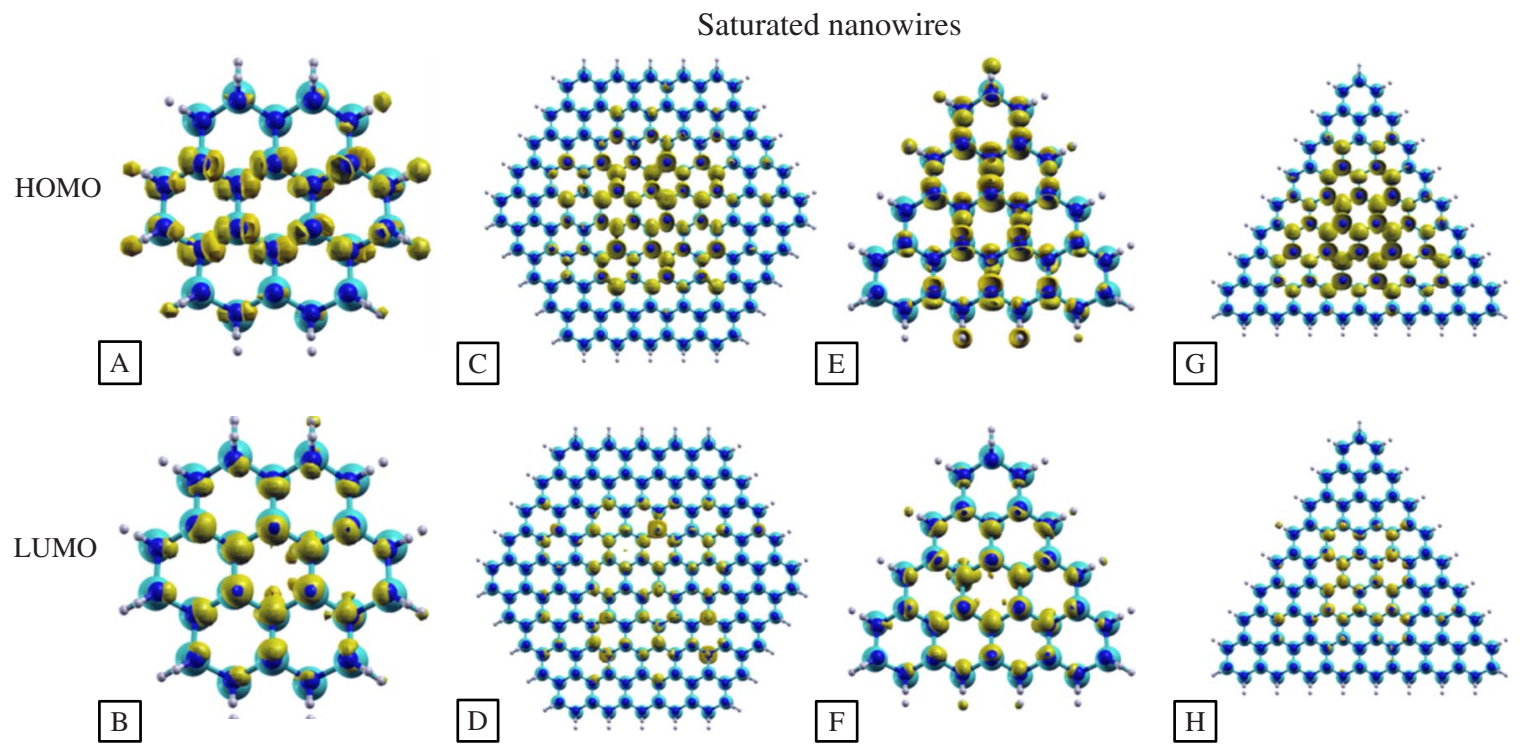

FIG. 7. (Color online) Spatial distribution of the HOMO and LUMO states at the gamma point for saturated nanowires, from DMOL ${ }^{3}$ calculations. The HOMO states (upper panel) are shown for hexagonal wires with diameters of (A) 9.5 (72 atoms) and (C) 28.6 (360 atoms) $\AA$, and triangular nanowires with diameters of (E) 12.7 (96 atoms) and (G) 25.5 (248 atoms). The LUMO states (lower panel) are shown for hexagonal wires with diameters of (B) 9.5 and (D) $28.6 \AA$, and triangular nanowires with diameters of (F) 12.7 and (H) $25.5 \AA$. Nitrogen and gallium atoms are dark (blue) and light (aqua) spheres, respectively, hydrogen atoms are represented by very small light gray spheres, and the orbitals are pale gray (yellow). 


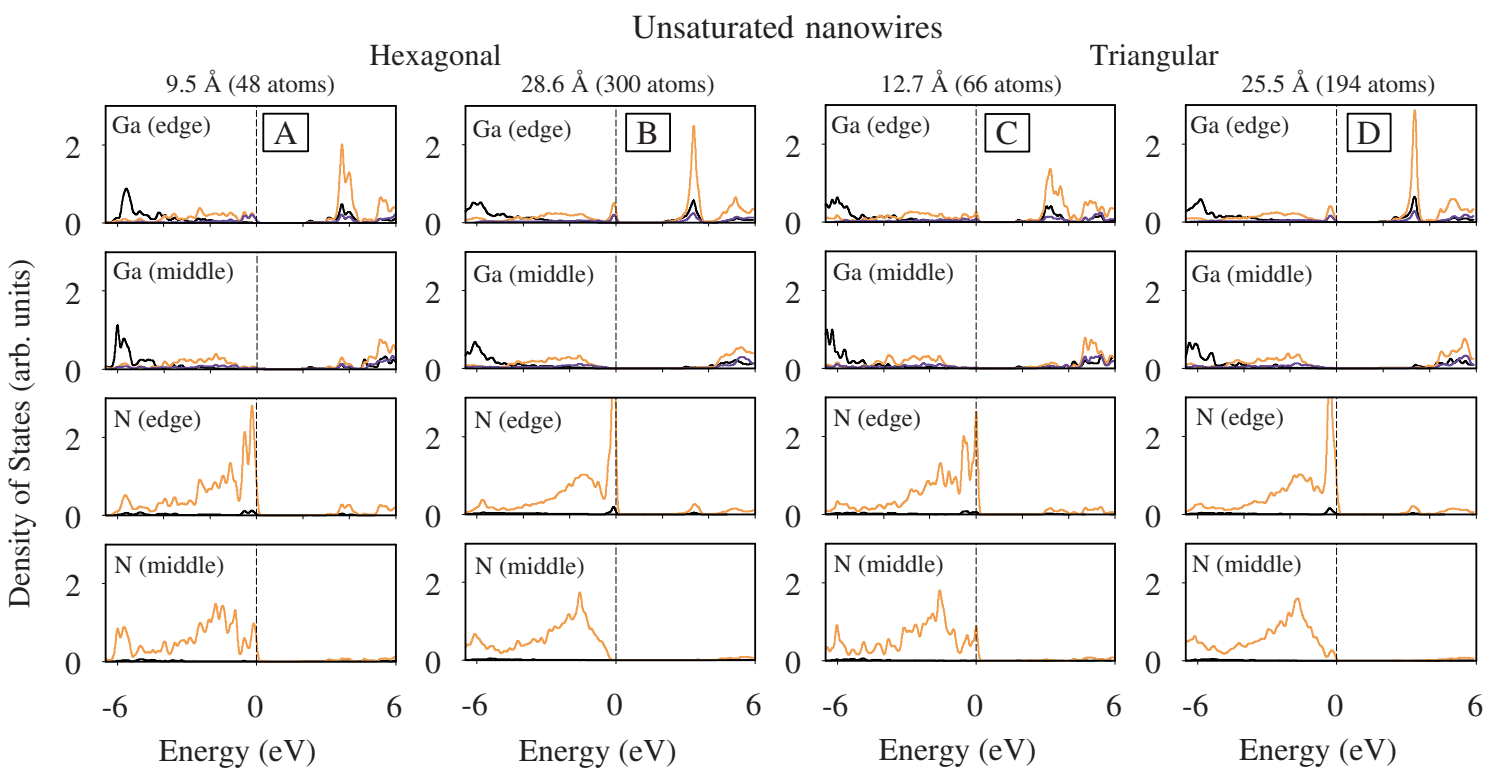

FIG. 8. (Color online) Partial density of states (PDOS) plots for unsaturated hexagonal nanowires with diameters of (A) 9.5 (48 atoms) and (B) 28.6 (300 atoms) Å, and triangular nanowires with diameters of (C) 12.7 (66 atoms) and (D) 25.5 (194 atoms) Å, calculated using the $\mathrm{DMOL}^{3}$ code. The black, light gray (orange), and dark gray (blue) lines correspond to $s-, p$ - and $d$-state contributions, respectively. The energy zero corresponds to the highest occupied state.

appear to be centered mainly on the gallium atoms, also with $p$ character. We investigate this in more detail by examining the atom-projected density of states, as discussed below. For the saturated nanowires illustrated in Fig. 7, the highest occupied and lowest unoccupied states have a significant weight distributed across the center of the nanowires. This is consistent for all nanowire diameters and for both shapes. For the smaller diameter nanowires, there can still be a vis- ible contribution toward the edge of the nanowires (a consequence of the small diameter), but this effect is much less for the larger diameter wires.

\section{Density of states}

In Figs. 8 and 9 we show the atom-projected density of states (PDOS). In particular, we consider gallium and nitrogen atoms at the edge of the nanowires and within the center

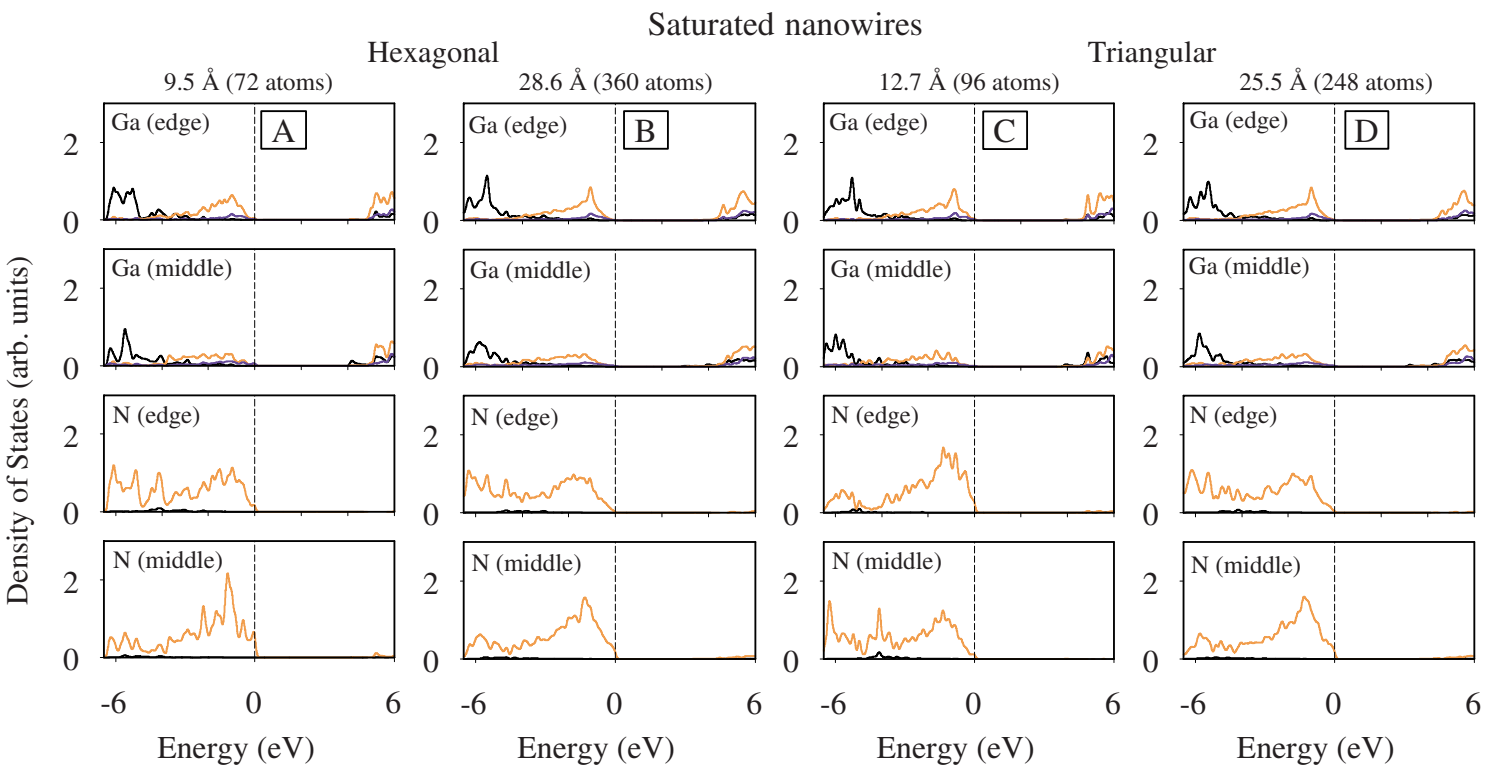

FIG. 9. (Color online) Partial density of states (PDOS) plots for saturated hexagonal nanowires with diameters of (A) 9.5 (72 atoms) and (B) 28.6 (360 atoms) $\AA$, and triangular nanowires with diameters of (C) 12.7 (96 atoms) and (D) 25.5 (248 atoms) $\AA$, calculated using the $\mathrm{DMOL}^{3}$ code. The black, light gray (orange), and dark gray (blue) lines correspond to $s-, p$ - and $d$-state contributions, respectively. The energy zero corresponds to the highest occupied state. 

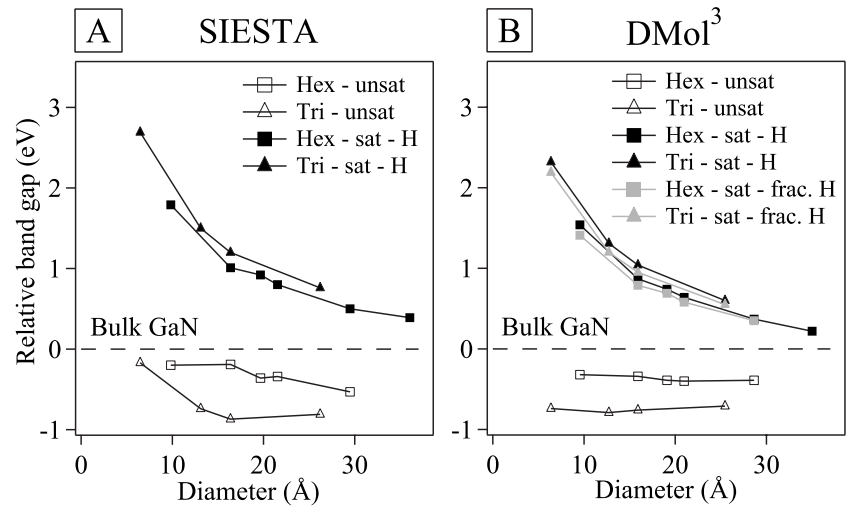

FIG. 10. Relative band gap as a function of nanowire diameter as obtained using the (A) SIESTA and (B) DMOL ${ }^{3}$ codes. Band gaps are relative to the calculated bulk GaN band gap. "Hex" and "Tri" represent "hexagonal" and "triangular," respectively, and "unsat," "sat," and "sat-frac. H" represent "unsaturated," "saturated," and "saturated with fractional charge hydrogen," respectively.

of the nanowires for comparison. We have also examined how the PDOS changes with the diameter of nanowires and the effect of saturating the dangling bonds with hydrogen. From Figs. 8(a) (small wire) and 8(b) (large wire) it can be seen that for a nitrogen and a gallium atom at the edge of an unsaturated nanowire, there are noticeable peaks at about $3.5 \mathrm{eV}$ for the gallium PDOS (contributed from the Ga $3 p$ orbital) and at about $-0.4 \mathrm{eV}$ for the nitrogen PDOS (contributed from the N $2 p$ orbital). These peaks are not present for the PDOS of gallium and nitrogen atoms in the center of unsaturated nanowires. This effect can be seen right across the range of nanowire diameters, and for both hexagonal and triangular [Figs. 8(c) and 8(d)] shaped nanowires. Thus, the gallium $3 p$ orbitals predominantly contribute to the edge states that form at and/or below the conduction band minimum and the nitrogen $2 p$ orbitals contribute to the edge states that form at and/or above the valence band maximum in the band structure plots shown previously in Fig. 4.

For a nitrogen and gallium atom at the edge of a saturated nanowire, Fig. 9 shows that there is little difference when compared to the PDOS of a similar atom in the center of a nanowire. The hydrogen atoms have the effect of stabilizing the bonding molecular orbitals and pushing the antibonding molecular orbitals up, which moves the edge states from the band gap (this stabilizing affect can also been seen in Fig. 5 in the band structure plots). This behavior occurs for both the small and large hexagonal [Figs. 9(a) and 9(b)] and triangular [Figs. 9(c) and 9(d)] shaped wires.

\section{Dependence of band gap on nanowire diameter}

The calculated band gaps as a function of nanowire diameter, for hexagonal and triangular nanowires, as obtained using SIESTA and $\mathrm{DMOL}^{3}$, are shown in Fig. 10. Using $\mathrm{DMOL}^{3}$, we also compare results obtained by saturating dangling bonds with hydrogen (one electron), and with "hydrogen" that has a fractional charge. The fractional charges are set such that they represent the environment an edge atom would have if it was in the bulk, namely, a charge of $0.75 e$ or $1.25 e$, depending on whether the dangling bond is from a nitrogen or gallium atom, respectively. This ensures that "perfect" covalent bonds are formed, as has been suggested for saturating dangling bonds of semiconductor compounds. ${ }^{61}$

The results in Fig. 10 show similar trends for both SIESTA and $\mathrm{DMOL}^{3}$. All saturated nanowires show a decrease in the band gap with increasing diameter, eventually approaching the bulk band gap values. For unsaturated nanowires, there is little change in the band gaps with increasing diameter, illustrating the influence of the localized edgelike dangling bond states in the band gap, mentioned previously. For the unsaturated nanowires, the band gaps of triangular nanowires are noticeably smaller than for hexagonal wires; however, for saturated wires, this trend is reversed, although the difference is much smaller. For a given effective wire diameter, the triangular and hexagonal nanowire calculations contain slightly different numbers of GaN units, so when we examine the band gap as a function of the number of atoms, the band gaps of the saturated hexagonal and triangular wires are extremely similar. The trend, however, for the unsaturated wires remains, with the band gap being consistently smaller for the triangular wires. This trend is perfectly understandable since the triangular wires are less stable, and the occupied edge-induced states are at a higher energy compared to the hexagonal wires (and thus the formation energy is higher, i.e., less stable, as well-see Sec III B 5).

For $\mathrm{DMOL}^{3}$, saturating with fractionally charged hydrogen shows similar results to saturation with hydrogen, for the band gap trends as a function of nanowire diameter. The band gaps, when saturated with fractionally charged hydrogen, are slightly less than when saturated with hydrogen. The orbital and PDOS plots when terminating with fractionally charged hydrogen atoms again show similar trends to those observed with hydrogen, although there are slight differences. So terminating with fractionally charged hydrogen atoms produces very similar behavior to terminating with hydrogen atoms. This similarity may be due to the fact that in all cases, there is always a pair of fractionally charged hydrogen atoms, which have charges of 0.75 and 1.25 , so the sum of the electrons is still 2, just like when terminating with a pair of standard hydrogen atoms. If the fractionally charged hydrogen atoms did not always occur in a pair, the results may be quite different.

We now examine the relationship between the relative band gap and nanowire diameter $(d)$, by fitting to the expression $\Delta E_{g}=A / d^{x}+c$. We plot the relative band gap versus $1 / d^{x}$ for saturated hexagonal [Fig. 11(a)] and triangular [Fig. 11(b)] nanowires from SIESTA and saturated hexagonal [Fig. 11(c)] and triangular [Fig. 11(d)] nanowires from $\mathrm{DMOL}^{3}$. From Fig. 11, overall there is a reasonable match to the linear relationship for the $1 / d^{x}$ plots, with the triangular shaped nanowires matching particularly well. For the hexagonal wires (and triangular, but to a lesser extent) with both $\mathrm{DMOL}^{3}$ and SIESTA [Figs. 11(a) and 11(b)], there is some deviation from the linear relationship at the larger diameters (smaller value of $\left.1 / d^{x}\right)$. Li and Wang ${ }^{62}$ performed DFT calculations, using the local-density approximation, to examine the $1 / d^{x}$ relationship for hexagonal $\mathrm{GaN}$ nanowires with diameters ranging from 11 to $32 \AA$, and reported the value of $x$ is 1.17 . The $x$ value for hexagonal wires from SIESTA is 1.17 and 

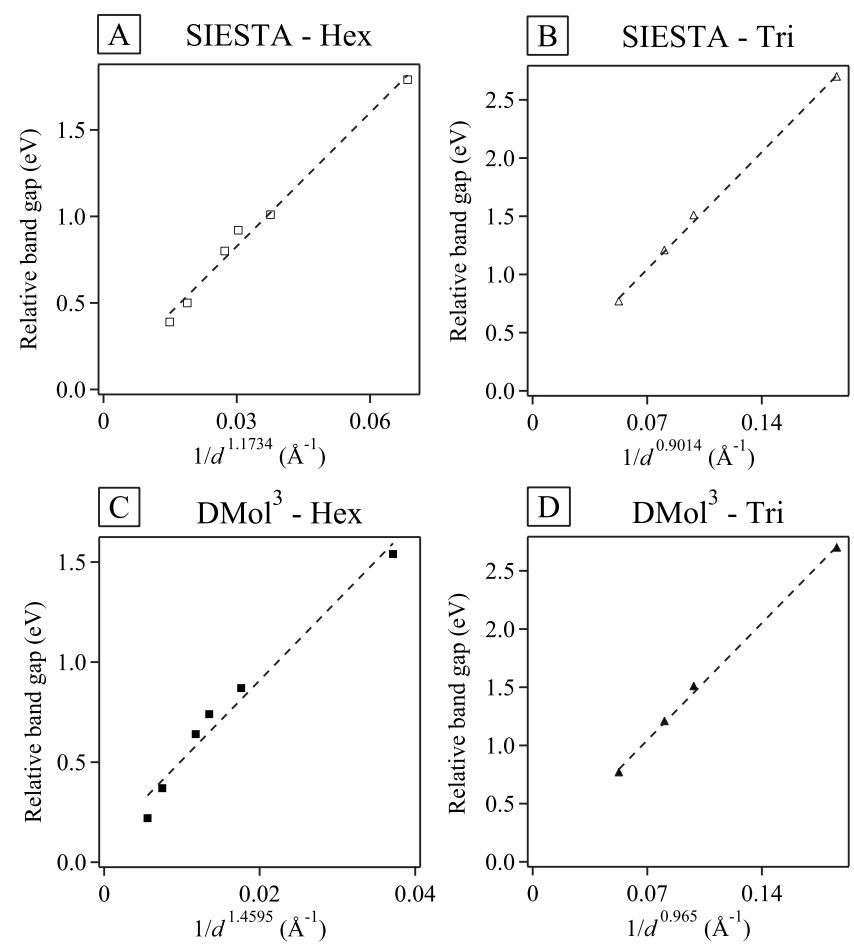

FIG. 11. Relative band gap as a function of $1 / d^{x}$ for saturated (A) hexagonal and (B) triangular nanowires with SIESTA, and saturated (C) hexagonal and (D) triangular nanowires with $\mathrm{DMOL}^{3}$, where $d$ is the nanowire diameter. The dashed lines indicate the linear regions for each of the data sets. "Hex" and "Tri" represent "hexagonal" and "triangular," respectively. Band gaps are relative to the calculated bulk GaN band gap.

from $\mathrm{DMOL}^{3}$ is 1.46 . The triangular wires have a smaller value of $x$, namely, 0.901 and 0.965 from SIESTA and $\mathrm{DMOL}^{3}$, respectively. Nanda et al. ${ }^{63}$ used a simple finite-depth squarewell model to study cylindrical wires, and reported the band gap is proportional to $1 / d^{2}$ (where $d$ is the diameter). We also plot the relative band gap versus $1 / d^{2}$ for saturated hexagonal [Fig. 12(a)] and triangular [Fig. 12(b)] nanowires. From Figs. 12(a) and 12(b), it can be seen that hexagonal nanowires with diameters ranging from 19.7 to $35.0 \AA$, and triangular nanowires with diameter ranging from 12.7 to $25.5 \AA$, appear to follow closely the $1 / d^{2}$ proportionality for the band gap change. Nanowires with smaller diameters deviate from this proportionality relationship. Schmidt et $a l .{ }^{31}$ reported a similar behavior for InP nanowires, where nanowires with diameters ranging from 18.0 to $21.3 \AA$ were studied. They suggested that for smaller diameter nanowires, the contribution of the nanowire surface to the electronic properties is not negligible, compared to the contribution for larger nanowires, leading to the deviation from the proportionality of the finite-depth square-well model. When we actually fit the larger hexagonal (19.7-35.0 $)$ and triangular (12.7-25.5 $\AA$ ) nanowires, we obtain $x$ values of 1.978 and 1.130 , respectively, using $\mathrm{DMOL}^{3}$, and 1.427 and 0.979 using SIESTA. For the hexagonal wires, taking into account only the larger diameter wires increases the $x$ value more toward the value of 2 obtained for the simple square-well model; the
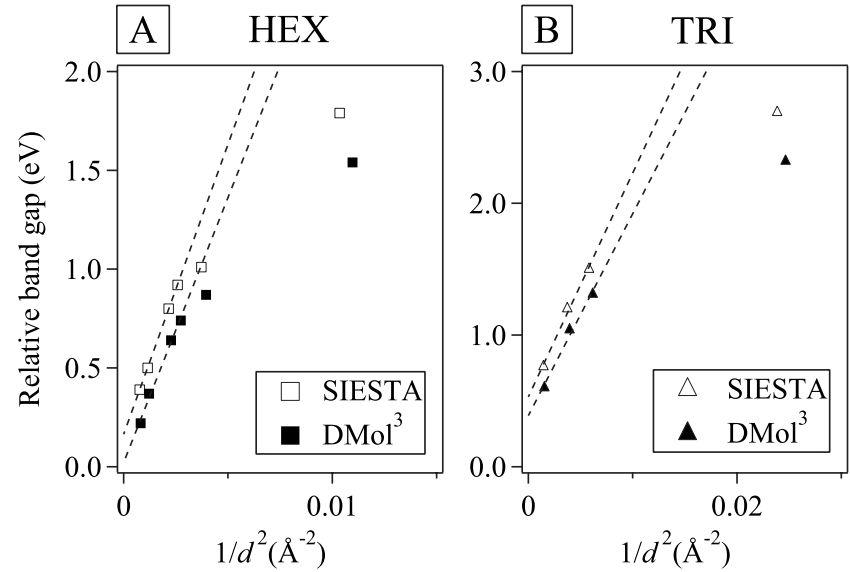

FIG. 12. Relative band gap as a function of $1 / d^{2}$ for saturated (A) hexagonal and (B) triangular nanowires, where $d$ is the nanowire diameter. The dashed lines indicate the linear regions for each of the data sets. "Hex" and "Tri" represent "hexagonal" and "triangular," respectively. Band gaps are relative to the calculated bulk GaN band gap.

corresponding values for the triangular wires only increase slightly.

The implications of these results are that there is a clear relationship between the nanowire diameter and its resulting band gap. This relationship can be exploited, as in a situation where a certain band gap for a particular application or device is required, in that we "simply" have to produce nanowires of correct corresponding diameter. It should of course be taken into consideration that band gaps calculated using DFT are systematically underestimated. Thus, in order to reproduce band gaps much closer to experimental values, more accurate calculations would be required, such as using the one-particle Green's function with screened Coulomb interaction $(\mathrm{GW})$ approach to predict quasiparticle band gaps. $^{24,64,65}$

We calculate the effective mass, with respect to the free electron mass $m_{0}$, around the CBM using the expression $\Delta E_{c}(\mathbf{k})=\hbar^{2} \mathbf{k}^{2} / 2 m_{c}^{*}$ where $m_{c}^{*}$ is the electron effective mass around the CBM. We fit a quadratic of the form $y=m x^{2}$, then solve $m=\hbar^{2} / 2 m_{c}^{*}$. For bulk GaN, we calculate $m_{c}^{*}$ to be $0.16 m_{0}$ and $0.27 m_{0}$ with SIESTA and $\mathrm{DMOL}^{3}$, respectively. These values are close to the reported experimental $m_{c}^{*}$ values of $0.20 \pm 0.02 m_{0}$ (Ref. 66) and $0.22 \pm 0.02 m_{0},{ }^{67}$ and the values from theoretical calculations of $0.13 m_{0},{ }^{68} 0.20 m_{0},{ }^{69,70}$ and $0.27 m_{0} .{ }^{71}$ We also examine the effect of nanowire diameter on the effective mass. For SIESTA, we calculate $m_{c}^{*}$ values of $0.48 m_{0}$ and $0.22 m_{0}$ for 9.5 and $28.6 \AA$ diameter hexagonal wires, and $0.41 m_{0}$ and $0.25 m_{0}$ for 12.7 and $25.5 \AA$ diameter triangular wires, respectively. A similar trend in effective mass is also observed for $\mathrm{DMOL}^{3}$ calculations, with $m_{c}^{*}$ decreasing as the nanowire diameter increases, approaching the bulk value. This trend is quite intuitive because as the diameter of the nanowire increases, the band gap gets smaller, which leads to an increase in the curvature of the CBM around the $\Gamma$ point and an increase of $m$ in the expression $m=\hbar^{2} / 2 m_{c}^{*}$, thus leading to a smaller effective mass. 

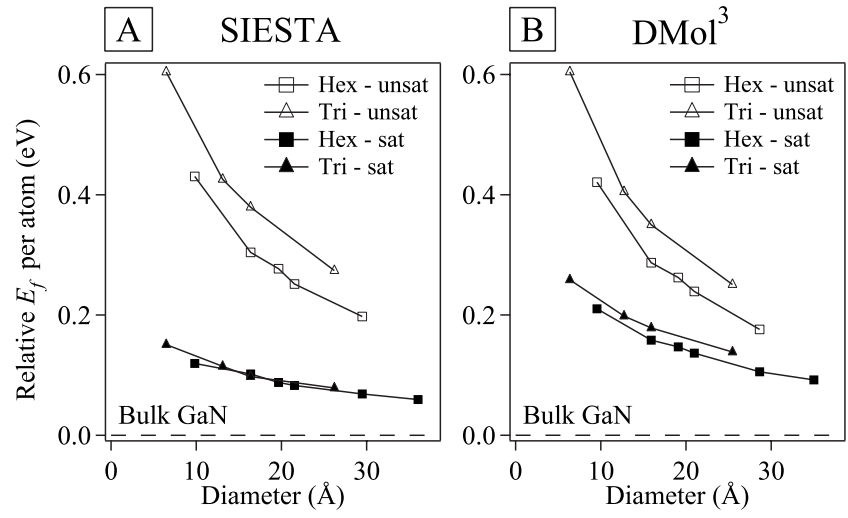

FIG. 13. Relative heat of formation (per atom) as a function of nanowire diameter for unsaturated and saturated nanowires in the [0001] direction, from (A) SIESTA and (B) DMOL ${ }^{3}$. Energies are relative to the heat of formation (per atom) of bulk GaN. "Hex" and "Tri" represent "hexagonal" and "triangular," respectively, and "unsat" and "sat" represent "unsaturated" and "saturated," respectively.

\section{Formation energies}

We now consider the heat of formation of hexagonal and triangular nanowires in the [0001] direction, for both unsaturated and saturated nanowires. For the unsaturated case, the heat of formation (per atom) is

$$
E_{f}=\left\{E(x \mathrm{GaN})-\left[x E(\mathrm{Ga})+\frac{x}{2} E\left(\mathrm{~N}_{2}\right)\right]\right\} /(2 x),
$$

where $E(x \mathrm{GaN})$ is the total energy of the relaxed unsaturated nanowire, $x$ is the number of GaN units in the supercell of the nanowire, $E(\mathrm{Ga})$ is the energy of a gallium atom obtained from the energy of bulk gallium metal, and $E\left(\mathrm{~N}_{2}\right)$ is the energy of a nitrogen molecule. We calculate the heat of formation (per atom) of saturated nanowires using the following expression:

$$
\begin{aligned}
E_{f}= & \left\{E[x(\mathrm{GaN}) y \mathrm{H}]-\left[x E(\mathrm{Ga})+\frac{x}{2} E\left(\mathrm{~N}_{2}\right)\right.\right. \\
& \left.\left.+\frac{y}{2} E\left(\mathrm{H}_{2}\right)\right]\right\} /(2 x+y),
\end{aligned}
$$

where $E[x(\mathrm{GaN}) y \mathrm{H}]$ is the total energy of the relaxed saturated nanowire, $y$ is the number of $\mathrm{H}$ atoms in the supercell of the saturated nanowire, and $E\left(\mathrm{H}_{2}\right)$ is the energy of a hydrogen molecule.

The heat of formation (per atom) for unsaturated and saturated nanowires as a function of nanowire diameter are shown in Fig. 13. From Fig. 13, it can be seen that as the diameter of the nanowires increases, the stability increases, as indicated by the lower heat of formation, approaching the stability of that found in bulk GaN. For results within each code, the hexagonal nanowires are slightly more stable than triangular nanowires of the same size. When all dangling bonds are saturated, the local bonding environment is very similar for the hexagonal and triangular wires, so we may expect that the formation energy per atom for saturated wires would also be very similar. For a given effective wire diameter, the triangular and hexagonal nanowire calculations contain slightly different numbers of $\mathrm{GaN}$ units, so when we examine the formation energy as a function of the number of atoms, the results for triangular and hexagonal wires are extremely similar. The hexagonal wires, however, are very slightly more stable. This can be explained in that for the same number of atoms, the triangular wires (assuming an equilateral triangular cross-section) have a slightly greater circumference (number of dangling bonds) compared to the corresponding hexagonal wires, and thus the triangular wires will always experience a slightly lesser degree of "real" GaN environment compared to the hexagonal wires.

We note that the surface formation energy of the unsaturated wires, $E_{f}^{\text {surf }}=\left(E_{\text {wire }}-x E_{\mathrm{GaN}}\right) / N_{\text {surf }}$, where $E_{\text {wire }}, E_{\mathrm{GaN}}$ and $N_{\text {surf }}$ are the total energies of the wire and a bulk GaN stoichiometric unit, and the number of surface atoms, respectively, exhibits a similar trend to the heat of formation. That is, a decrease with increasing wire diameter and a greater stability (lower surface formation energy) for the hexagonal wires. For example, using $\mathrm{DMOL}^{3}$ values of $0.56,0.52,0.51$, 0.50 , and $0.49 \mathrm{eV}$ are obtained for the hexagonal 9.5, 15.9, 19.7, 21.0, and $28.6 \AA$ diameter nanowires, respectively. For the triangular wires, the surface formation energies are 0.98 , $0.56,0.54$, and $0.50 \mathrm{eV}$ for the $6.3,12.7,15.9$, and $25.5 \AA$ diameter nanowires, respectively. Similar trends with regard to shape and a decrease in heat of formation with increasing wire diameter are obtained for saturated wires, although saturating nanowires with hydrogen stabilize the nanowires, as indicated by the lower heat of formation for the same size wires. Once again, the SIESTA and $\mathrm{DMOL}^{3}$ codes produce similar results.

\section{CONCLUSIONS}

We have examined the atomic and electronic structures and stability of hexagonal and triangular $\mathrm{GaN}$ nanowires in the [0001] growth direction, and the influence of saturating dangling bonds for a wide range of diameters $(8-35 \AA)$. The atomic relaxations of the nanowires exhibit similar bond length and bond angle changes across the range of nanowire diameters and shapes examined. For unsaturated wires, we found edge-induced dangling bond states in the region of the band gap. The positions of these states remain rather constant with varying diameter size for both the hexagonal and triangular wires. Saturating these dangling bonds with hydrogen removes the edge states from the band gap, such that the band gap decreases with increasing nanowire diameter. For the unsaturated nanowires, from consideration of the spatial distribution of the highest occupied state and lowest unoccupied state, and atom-projected density of states, we found that the nitrogen $2 p$ orbitals contribute to the edge states at the valence band maximum and gallium $3 p$ orbitals predominantly contribute to edge states at the conduction band minimum. We calculated the effective electron mass for hexagonal and triangular wires and found that it decreases with increasing diameter, approaching that of the bulk value. We examined the relationship between nanowire diameter $(d)$ 
and band gap by fitting a $1 / d^{x}$ relationship, finding the $x$ value for triangular wires is smaller than that for hexagonal wires. When plotted against a $1 / d^{2}$ relationship, there is a close match for large diameter nanowires; however, smaller diameter nanowires deviate from this expression. The heat of formation of the nanowires decreases with increasing diameter, approaching that of the bulk value.

\section{ACKNOWLEDGMENTS}

We gratefully acknowledge the Australian Partnership for Advanced Computing National Facility and the Australian Centre for Advanced Computing and Communications. This work was supported by the Australian Research Council. J.D.G. would like to thank the Government of Western Australia. *carter@physics.usyd.edu.au

${ }^{1}$ J. V. Barth, G. Costantini, and K. Kern, Nature (London) 437, 671 (2005).

${ }^{2}$ C. M. Lieber and Z. L. Wang, MRS Bull. 32, 99 (2007).

${ }^{3}$ J. C. Johnson, H.-J. Choi, K. P. Knutsen, R. D. Schaller, P. Yang, and R. J. Saykally, Nat. Mater. 1, 106 (2002).

${ }^{4}$ J. Goldberger, R. He, Y. Zhang, S. Lee, H. Yan, H.-J. Choi, and P. Yang, Nature (London) 422, 599 (2003).

${ }^{5}$ J. Hu, Y. Bando, D. Golberg, and Q. Liu, Angew. Chem., Int. Ed. 42, 3493 (2003).

${ }^{6}$ J.-R. Kim, H. M. So, J. W. Park, J.-J. Kim, J. Kim, C. J. Lee, and S. C. Lyu, Appl. Phys. Lett. 80, 3548 (2002).

${ }^{7}$ G. Kipshidze, B. Yavich, A. Chandolu, J. Yun, V. Kuryatkov, I. Ahmad, D. Aurongzeb, M. Holtz, and H. Temkin, Appl. Phys. Lett. 86, 033104 (2005).

${ }^{8}$ C.-C. Chen and C.-C. Yeh, Adv. Mater. (Weinheim, Ger.) 12, 738 (2000).

${ }^{9}$ C. Y. Nam, D. Tham, and J. E. Fischer, Appl. Phys. Lett. 85, 5676 (2004).

${ }^{10}$ B. S. Simpkins, L. M. Ericson, R. M. Stroud, K. A. Pettigrew, and P. E. Pehrsson, J. Cryst. Growth 290, 115 (2006).

${ }^{11}$ B.-S. Xu, L.-Y. Zhai, J. Liang, S.-F. Ma, H.-S. Jia, and X.-G. Liu, J. Cryst. Growth 291, 34 (2006).

${ }^{12}$ Y.-K. Byeun, K.-S. Han, and S.-C. Choi, J. Electroceram. 17, 903 (2006).

${ }^{13}$ S. Dhara, A. Datta, C. T. Wu, Z. H. Lan, K. H. Chen, Y. L. Wang, C. W. Hsu, C. H. Shen, L. C. Chen, and C. C. Chen, Appl. Phys. Lett. 84, 5473 (2004).

${ }^{14}$ H. Y. Peng, N. Wang, X. T. Zhou, Y. F. Zheng, C. S. Lee, and S. T. Lee, Chem. Phys. Lett. 359, 241 (2002).

${ }^{15}$ T. Kuykendall, P. J. Pauzauskie, Y. Zhang, J. Goldberger, D. Sirbuly, J. Denlinger, and P. Yang, Nat. Mater. 3, 524 (2004).

${ }^{16}$ G. Cheng, A. Kolmakov, Y. Zhang, M. Moskovits, R. Munden, M. A. Reed, G. Wang, D. Moses, and J. Zhang, Appl. Phys. Lett. 83, 1578 (2003).

${ }^{17}$ F. L. Deepak, P. V. Vanitha, A. Govindaraj, and C. N. R. Rao, Chem. Phys. Lett. 374, 314 (2003).

${ }^{18}$ D. S. Han, J. Park, K. W. Rhie, S. Kim, and J. Chang, Appl. Phys. Lett. 86, 032506 (2005).

${ }^{19}$ H.-J. Choi, H.-K. Seong, J. Chang, K.-I. Lee, Y.-J. Park, J.-J. Kim, S.-K. Lee, R. He, T. Kuykendall, and P. Yang, Adv. Mater. (Weinheim, Ger.) 17, 1351 (2005).

${ }^{20}$ M.-H. Tsai, Z.-F. Jhang, J.-Y. Jiang, Y.-H. Tang, and L. W. Tu, Appl. Phys. Lett. 89, 203101 (2006).

${ }^{21}$ A. Gulans and I. Tale, Phys. Status Solidi C 4, 1197 (2007).

${ }^{22}$ Q. Wang, Q. Sun, and P. Jena, Phys. Rev. Lett. 95, 167202 (2005).

${ }^{23}$ Q. Wang, Q. Sun, P. Jena, and Y. Kawazoe, Nano Lett. 5, 1587
(2005).

${ }^{24}$ X. Zhao, C. M. Wei, L. Yang, and M. Y. Chou, Phys. Rev. Lett. 92, 236805 (2004).

${ }^{25}$ Y. Zheng, C. Rivas, R. Lake, K. Alam, T. B. Boykin, and G. Klimeck, IEEE Trans. Electron Devices 52, 1097 (2005).

${ }^{26}$ J. Li and A. J. Freeman, Phys. Rev. B 74, 075333 (2006).

${ }^{27}$ R. Kagimura, R. W. Nunes, and H. Chacham, Phys. Rev. Lett. 98, 026801 (2007).

${ }^{28}$ W. Fan, H. Xu, A. L. Rosa, T. Frauenheim, and R. Q. Zhang, Phys. Rev. B 76, 073302 (2007).

${ }^{29}$ M. Zhao, Y. Xia, X. Liu, Z. Tan, B. Huang, C. Song, and L. Mei, J. Phys. Chem. B 110, 8764 (2006).

${ }^{30}$ R. Leitsmann and F. Bechstedt, J. Appl. Phys. 102, 063528 (2007).

${ }^{31}$ T. M. Schmidt, R. H. Miwa, P. Venezuela, and A. Fazzio, Phys. Rev. B 72, 193404 (2005).

${ }^{32}$ P. Ordejon, E. Artacho, and J. M. Soler, Phys. Rev. B 53, R10441 (1996).

${ }^{33}$ J. M. Soler, E. Artacho, J. D. Gale, A. Garcia, J. Junquera, P. Ordejon, and D. Sanchez-Portal, J. Phys.: Condens. Matter 14, 2745 (2002).

${ }^{34}$ B. Delley, J. Chem. Phys. 92, 508 (1990).

${ }^{35}$ B. Delley, J. Chem. Phys. 113, 7756 (2000).

${ }^{36}$ J. P. Perdew, K. Burke, and M. Ernzerhof, Phys. Rev. Lett. 77, 3865 (1996).

${ }^{37}$ N. Troullier and J. L. Martins, Phys. Rev. B 43, 1993 (1991).

${ }^{38}$ H. J. Monkhorst and J. D. Pack, Phys. Rev. B 13, 5188 (1976).

${ }^{39}$ B. Delley, Phys. Rev. B 66, 155125 (2002).

${ }^{40}$ S. D. Fleming and A. L. Rohl, Z. Kristallogr. 220, 580 (2005).

${ }^{41}$ D. R. Lide, CRC Handbook of Chemistry and Physics, 81st ed. (CRC, London, 2003).

${ }^{42}$ C. Stampfl and C. G. Van de Walle, Phys. Rev. B 59, 5521 (1999).

${ }^{43}$ C. S. Barrett and F. J. Spooner, Nature (London) 207, 1382 (1965).

${ }^{44}$ T. Kenichi, K. Kazuaki, and A. Masao, Phys. Rev. B 58, 2482 (1998).

${ }^{45}$ M. Fuchs, J. L. F. Da Silva, C. Stampfl, J. Neugebauer, and M. Scheffler, Phys. Rev. B 65, 245212 (2002).

${ }^{46}$ X. G. Gong, G. L. Chiarotti, M. Parrinello, and E. Tosatti, Phys. Rev. B 43, 14277 (1991).

${ }^{47}$ J. Junquera, O. Paz, D. Sanchez-Portal, and E. Artacho, Phys. Rev. B 64, 235111 (2001).

${ }^{48}$ H. Schulz and K. H. Thiemann, Solid State Commun. 23, 815 (1977).

${ }^{49}$ H. Xia, Q. Xia, and A. L. Ruoff, Phys. Rev. B 47, 12925 (1993).

${ }^{50}$ P. Perlin, C. Jauberthie-Carillon, J. P. Itie, A. San Miguel, I. Grzegory, and A. Polian, Phys. Rev. B 45, 83 (1992). 
${ }^{51}$ B. Monemar, Phys. Rev. B 10, 676 (1974).

${ }^{52}$ B. Paulus, F. Shi, and H. Stoll, J. Phys.: Condens. Matter 9, 2745 (1997).

${ }^{53}$ O. Kubaschewski and C. B. Alcock, Metallurgical Thermochemistry (Permagon, Oxford, 1979).

${ }^{54}$ I. Arslan, S. Ogut, P. D. Nellist, and N. D. Browning, Micron 34, 255 (2003).

${ }^{55}$ S. Strite, J. Ruan, Z. Li, A. Salvador, H. Chen, D. J. Smith, W. J. Choyke, and H. Morkoç, J. Vac. Sci. Technol. B 9, 1924 (1991).

${ }^{56}$ C. A. Morrison and M. M. Siddick, Chem.-Eur. J. 9, 628 (2003).

${ }^{57}$ S. D. Hersee, X. Sun, and X. Wang, Nano Lett. 6, 1808 (2006).

${ }^{58}$ T. Kuykendall, P. J. Pauzauskie, S. Lee, Y. Zhang, J. Goldberger, and P. Yang, Nano Lett. 3, 1063 (2003).

${ }^{59}$ S. Gradecak, F. Qian, Y. Li, H.-G. Park, and C. M. Lieber, Appl. Phys. Lett. 87, 173111 (2005).

${ }^{60}$ J. E. Northrup and J. Neugebauer, Phys. Rev. B 53, R10477 (1996).
${ }^{61}$ K. Shiraishi, J. Phys. Soc. Jpn. 59, 3455 (1990).

${ }^{62}$ J. Li and L. W. Wang, Phys. Rev. B 72, 125325 (2005).

${ }^{63}$ K. K. Nanda, F. E. Kruis, and H. Fissan, Nano Lett. 1, 605 (2001).

${ }^{64}$ M. S. Hybertsen and S. G. Louie, Phys. Rev. B 34, 5390 (1986).

${ }^{65}$ M. Shishkin and G. Kresse, Phys. Rev. B 75, 235102 (2007).

${ }^{66}$ A. S. Barker, Jr. and M. Ilegems, Phys. Rev. B 7, 743 (1973).

${ }^{67}$ P. Perlin, L. Litwin-Staszewska, B. Suchanek, W. Knap, J. Camassel, T. Suski, R. Piotrzkowski, I. Grzegory, and S. Porowski, Appl. Phys. Lett. 68, 1114 (1996).

${ }^{68}$ W. J. Fan, M. F. Li, T. C. Chong, and J. B. Xia, J. Appl. Phys. 79, 188 (1996).

${ }^{69}$ K. Miwa and A. Fukumoto, Phys. Rev. B 48, 7897 (1993).

${ }^{70}$ M. Suzuki, T. Uenoyama, and A. Yanase, Phys. Rev. B 52, 8132 (1995).

${ }^{71}$ Y.-N. Xu and W. Y. Ching, Phys. Rev. B 48, 4335 (1993). 